\title{
Dynamical Behavior in the Vicinity of a Circular Anisotropic Ring
}

\author{
Nour-Eddine Najid*, Mohammed Zegoumou and El Haj Elourabi
}

Equipe Mécanique Statistique et Astronomie Laboratoire de Physique Théorique et Appliquée Université Hassan II Ain Chock- Faculté des Sciences Ain Chock BP 5366, Maârif Casablanca, Morocco

\begin{abstract}
Motivated by the variety of rings around planets in our solar system, and arguably in other planetary systems as well, we will analytically treat the venerable problem of investigating the potential of a massive annular distribution. In a first approximation, we can consider the mass distribution as homogenous. Actually, a more realistic model should take into account the non-isotropy of density.In this work, we establish an analytical elliptic form of the potential generated by an anisotropic matter distribution. The study of the dynamical behavior is performed within the Hamiltonian formulation, which allows us to derive some orbits of the test particle.
\end{abstract}

Keywords: Potential - Anisotropic Distribution-Rings- Elliptic Integrals.

\section{INTRODUCTION}

The solar system is a large area with various kinds of celestial bodies; elongated, circular, elliptical or strongly irregular ones.

Their gravitational behaviour gives us much information. The relativistic studies allowed us to valid some aspect of this theory [1] or to detect relativistic effect about the element of orbits [2], or the increase of accuracy of the two body problem in the frame of general relativity [3]. The extra solar planets are studied too in [4-6] and [7]. The effect of atmosphere, potential type or gravitational radiation about orbits are studied in [8,9], and [10]. Some other publications [11-14] are devoted to studies of rings around planets or even sun.

The discoveries of binary asteroids, the mission to Saturn rings gave a new interest in potential calculation. This subject is a new/old field of research, $[15,16]$. In the literature we find studies like in Riaguas et al. [17] in which they estimated the potential generated by a homogeneous straight segment. Elipe and Lara [18] described the motion around Eros 433 with the same homogeneous model.

A harmonic polyhedron was used by Werner and Scheeres for 4769 Castalia $[19,20]$. Ellipsoids, material points and a segment of double material were used by Bartczak and Breiter [21] and Bartczak et al. [22]. In our laboratory we used a new idea in a previous work [23] by studying the potential generated by a massive straight segment with a parabolic profile of mass distribution. About rings, Harry et al. [24] calculated the potential due to uniform disk and

*Address correspondence to this author at the Equipe Mécanique Statistique et Astronomie Laboratoire de Physique Théorique et Appliquée Université Hassan II Ain Chock- Faculté des Sciences Ain Chock BP 5366, Maârif Casablanca, Morocco; Tel: 00212522230680; Fax: 00212522230674;

E-mail: ne.najid@gmail.com deduced that of a homogeneous ring. Broucke et al. [25] established the potential of a homogeneous circular ring and studied the properties and perturbations of orbits around a central planet surrounded by that ring. Fred et al. [26] established the expressions for both the potential and the field of a disk. They suggested the formulas of a ring. In our present work we propose a new idea by using an anisotropic mass density for a circular ring. In section 2 we will establish the integral expression of the potential generated by an anisotropic ring in space. In section 3 we give the analytical expression of the potential in the plane perpendicular to the ring in terms of the complete elliptical integral of the first kind $\mathrm{K}(\mathrm{k})$ and second kind $\mathrm{E}(\mathrm{k})$. We plot the level curves of this potential. In section 4 we give the set of differential equations by using the Hamiltonian formulation. Finally, in section 5 we solve the differential equations of motion and discuss different dynamical states of the test particle around the ring, we draw some orbits and discuss their gravitational features.

\section{INTEGRAL EXPRESSION OF THE POTENTIAL}

We consider a circular ring of radius a and total mass $\mathrm{M}$, located in the (xoy) plane (Fig. 1).

The density of the ring is given by:

$$
\lambda(\theta)=\lambda_{0}\left(1+b \cos ^{2} \frac{\theta}{2}\right)
$$

In which $\lambda_{0}$ and $b$ are positive constants.

The total mass of the ring is given by: $M=a \lambda_{0} \pi(b+2)$ and $b>-2$

If $b>0$ the line density is (Fig. 2):

- Maximum for $\theta=0: \lambda=\lambda_{0}(1+b)$ 


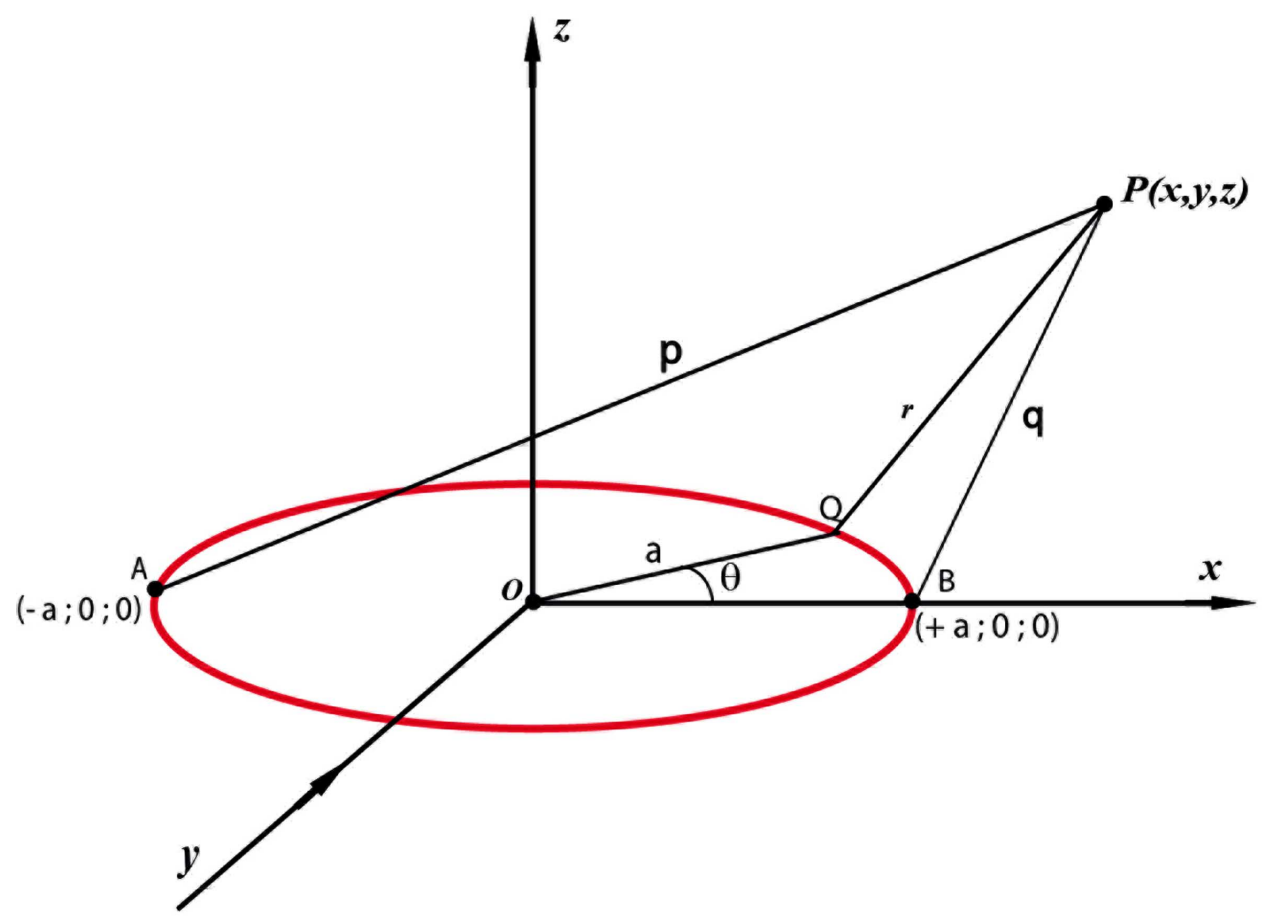

Fig. (1). Ring in the plane (xoy).
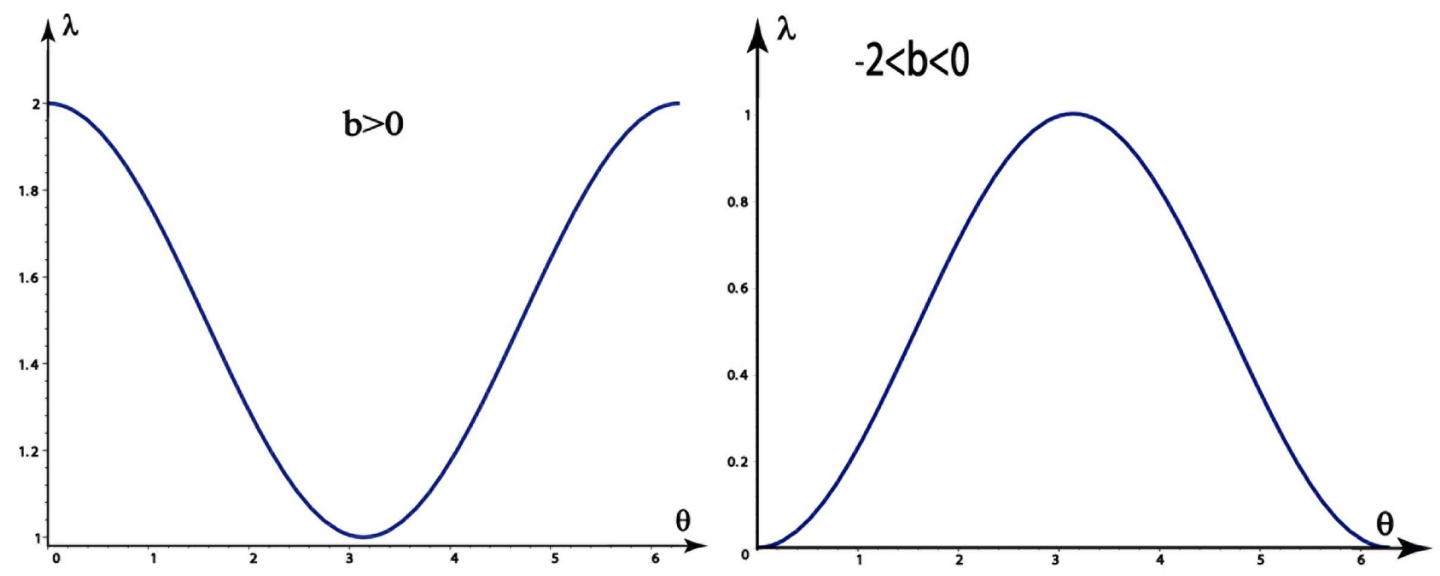

Fig. (2). Variation of the density.

- Minimum for $\theta=\pi: \lambda=\lambda_{0}$

If $-2<b<0$ the line density is (Fig. 2):

- Maximum for $\theta=\pi: \lambda=\lambda_{0}$

- Minimum for $\theta=0: \lambda=\lambda_{0}(1+b)$

Since the distribution is inhomogeneous its center of mass is not in O. By symmetry, the center of mass is at the position:

$\left(x_{G}=\frac{a b}{2(b+2)} ; y_{G}=0\right)$

The gravitational potential generated by the ring at a point $P$ $(\mathrm{x}, \mathrm{y}, \mathrm{z})$ is expressed by (Fig. 1):

$r$ is the distance between the element dm centered at Q, (Fig. 1) and $P$.

$$
r=Q P=\sqrt{(x-a \cos \theta)^{2}+(y-a \sin \theta)^{2}+z^{2}}
$$

We introduce two new auxiliary functions $\mathrm{p}$ and $\mathrm{q}$ defined by:

$\checkmark \quad \mathrm{p}$ is the largest distance between $\mathrm{P}$ and the ring given by:

$p=\sqrt{(x+a)^{2}+y^{2}+z^{2}}$

$\checkmark \quad \mathrm{q}$ is the smallest distance between $\mathrm{P}$ and the ring given by:

$q=\sqrt{(x-a)^{2}+y^{2}+z^{2}}$

$U(P)=-G \int_{r i n g} \frac{d m}{r}$

\section{- Expression of $r$ :}

The substitution of $\mathrm{p}$ and $\mathrm{q}$ in (4) gives:

$r=Q P=\sqrt{\frac{p^{2}+q^{2}}{2}-\frac{p^{2}-q^{2}}{2} \cos \theta-2 \text { ay } \sin \theta}$ 


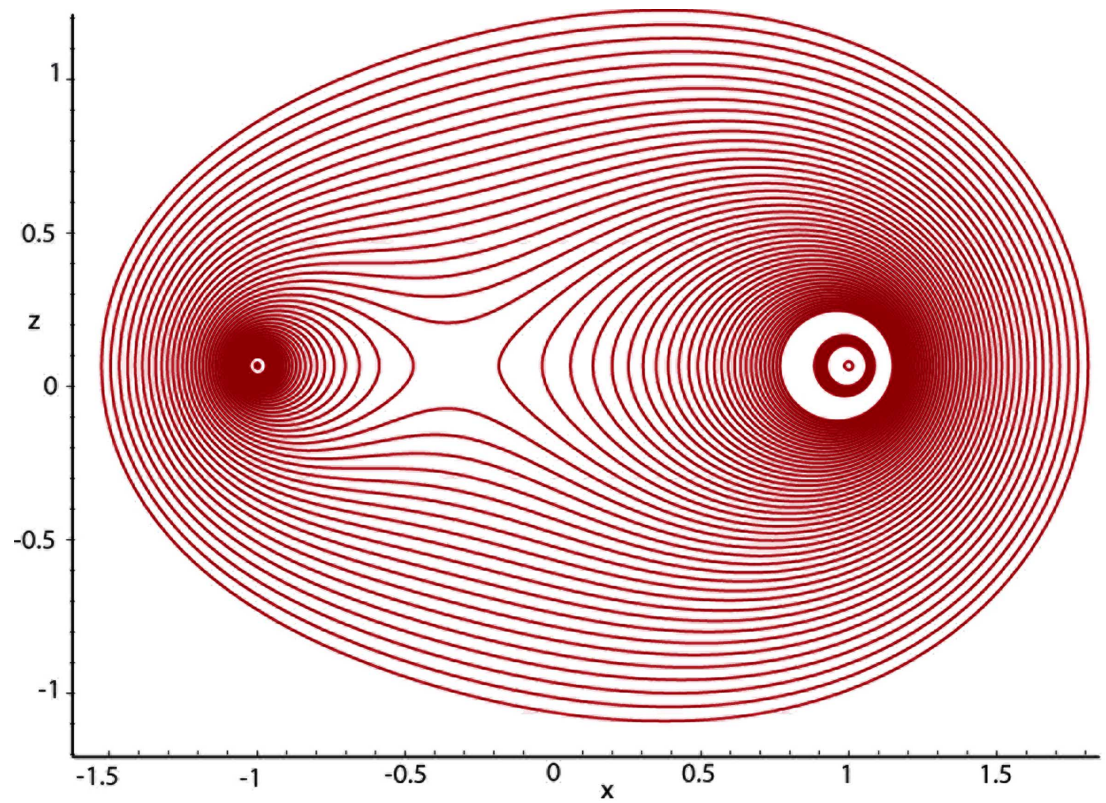

Fig. (3). Level cures of the potential function $U=U(x, z)$.

And:

$r=\sqrt{p^{2}\left(1-k^{2} \cos ^{2} \frac{\theta}{2}\right)-2 a y \sin \theta}$

With: $k^{2}=1-\frac{q^{2}}{p^{2}}<1$

Expression of dm:

$U=-G a \lambda_{0} \int_{0}^{2 \pi} \frac{1+b \cos ^{2} \frac{\theta}{2}}{\sqrt{\frac{p^{2}+q^{2}}{2}-\frac{p^{2}-q^{2}}{2} \cos \theta-2 a y \sin \theta}} d \theta$

Substituting the expressions (6) and (7) in (3), the potential generated by the ring is:

$U=-G a \lambda \int_{0}^{2 \pi} \frac{1+b \cos ^{2} \frac{\theta}{2}}{\sqrt{\frac{p^{2}+q^{2}}{2}-\frac{p^{2}-q^{2}}{2} \cos \theta-2 a y \sin \theta}} d \theta$

If we put $2 \varphi=\pi-\theta$

The expression (6) becomes:

$r=\sqrt{p^{2}\left(1-k^{2} \sin ^{2} \varphi\right)-2 a y \sin (2 \varphi)}$

Finally, the gravitational potential generated by the inhomogeneous ring at any point $\mathrm{P}$ in space is given by:

$$
U=-2 G a \lambda_{0} \int_{-\frac{\pi}{2}}^{\frac{\pi}{2}} \frac{\left(1+b \sin ^{2} \varphi\right) \cdot d \varphi}{\sqrt{p^{2}\left(1-k^{2} \sin ^{2} \varphi\right)-2 a y \sin 2 \varphi}}
$$

\section{ANALYTICAL EXPRESSION OF THE POTENTIAL IN (XOZ) PLANE}

In this section we will derive the analytical expression of the potential given by (9), in the (xoz) plane:

$$
U=-\frac{2 G a \lambda_{0}}{p} \int_{-\frac{\pi}{2}}^{\frac{\pi}{2}} \frac{\left(1+b \sin ^{2} \varphi\right) \cdot d \varphi}{\sqrt{\left(1-k^{2} \sin ^{2} \varphi\right)}}
$$

$$
\begin{aligned}
& U=-\frac{4 G \cdot a \lambda_{0}}{p} \int_{0}^{\frac{\pi}{2}} \frac{1+b \sin ^{2} \varphi}{\sqrt{\left(1-k^{2} \sin ^{2}(\varphi)\right)}} d \varphi \\
& U=-\frac{4 G a \lambda_{0}}{p} \int_{0}^{\frac{\pi}{2}} \frac{d \varphi}{\sqrt{\left(1-k^{2} \sin ^{2} \varphi\right)}}+\frac{4 G a b \lambda_{0}}{p k^{2}} \int_{0}^{\frac{\pi}{2}} \frac{-k^{2} \sin ^{2} \varphi}{\sqrt{\left(1-k^{2} \sin ^{2} \varphi\right)}} d \varphi
\end{aligned}
$$

$$
U=-\frac{4 G a \lambda_{0}}{p} K(k)+\frac{4 G a \lambda_{0} b}{p \cdot k^{2}}\left[-\int_{0}^{\frac{\pi}{2}} \frac{1}{\sqrt{\left(1-k^{2} \sin ^{2}(\varphi)\right)}} d \varphi+\int_{0}^{\frac{\pi}{2}} \frac{1-k^{2} \times \sin ^{2}(\varphi)}{\sqrt{\left(1-k^{2} \sin ^{2}(\varphi)\right)}} d \varphi\right]
$$

Where $\mathrm{K}(\mathrm{k})$ is the complete elliptic integral of first kind [27].

$$
\begin{aligned}
& U=-\frac{4 G a \lambda_{0}}{p} K(k)-\frac{4 G a \lambda_{0} b}{p \cdot k^{2}}\left[K(k)-\int_{0}^{\frac{\pi}{2}} \sqrt{1-k^{2} \sin ^{2}(\varphi)} d \varphi\right] \\
& U=-\frac{4 G a \lambda_{0}}{p} K(k)-\frac{4 G a \lambda_{0} b}{p \cdot k^{2}}[K(k)-E(k)]
\end{aligned}
$$

Where, $\mathrm{E}(\mathrm{k})$ is the complete elliptic integral of second kind.

Finally we reach the analytical expression of the gravitational potential generated by the anisotropic circular ring in the $(\mathrm{xoz})$ plane.

$$
U=-\frac{4 G \lambda_{0} a}{p k^{2}}\left[\left(k^{2}+b\right) K(k)-b E(k)\right]
$$

The expression (11) represents the analytical form of the potential.

The effect of non-uniform distribution is owing to $b$.

For $b=0$ we find the particular case of a homogeneous circular ring studied by Broucke et al. in [11].

-Fig. (3) shows the plot of equipotential contours, the analysis of this figure shows the existence of a hyperbolically unstable point corresponding to maximum potential. This maximum corresponds to a maximum of the density. 


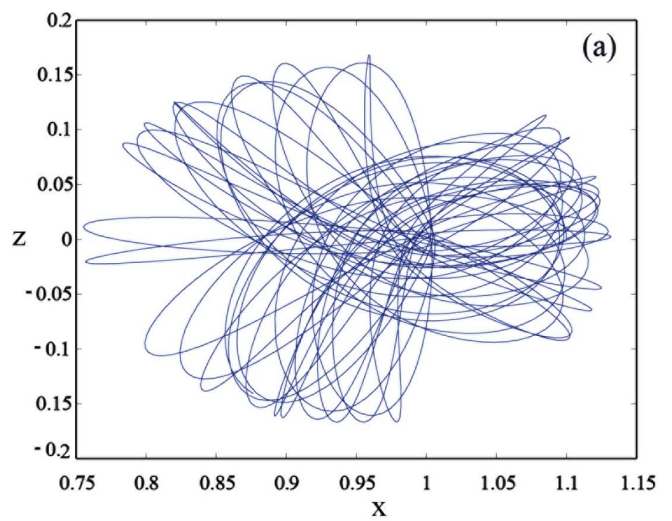

Fig. (4). Curve in (xoz) plane for $b=0: v_{0 z}=2$ and $v_{0 z}=3$.

\section{DYNAMICAL STUDY}

We study the dynamical behavior of a test particle, with unit mass, in the field of the inhomogeneous ring. The Hamiltonian of the test particle is given by:

$H=\frac{1}{2}\left(p_{1}^{2}+p_{2}^{2}\right)-\frac{4 G \lambda_{0} a K(k)}{p}+\frac{4 G \lambda_{0} a b(E(k)-K(k))}{p k^{2}}$

The equations of motion are given by:

$$
\begin{aligned}
& \left\{\begin{array}{l}
\ddot{x}=-\frac{\partial U}{\partial x}=-4 G \lambda_{0} a\left\{\left[E(k) \frac{\partial}{\partial x}\left(\frac{b}{p k^{2}}\right)+\left(\frac{b}{p k^{2}}\right)\left(\frac{E(k)-K(k)}{k}\right) \frac{\partial k}{\partial x}\right]\right. \\
\left.-K(k) \frac{\partial}{\partial x}\left(\frac{1}{p}+\frac{b}{p k^{2}}\right)-\left(\frac{1}{p}+\frac{b}{p k^{2}}\right)\left(\frac{E(k)-\left(1-k^{2}\right) K(k)}{k\left(1-k^{2}\right)}\right) \frac{\partial k}{\partial x}\right\}
\end{array}\right. \\
& \left\{\begin{array}{l}
\ddot{z}=-\frac{\partial U}{\partial z}=-4 G \lambda_{0} a\left\{\left[E(k) \frac{\partial}{\partial z}\left(\frac{b}{p k^{2}}\right)+\left(\frac{b}{p k^{2}}\right)\left(\frac{E(k)-K(k)}{k}\right) \frac{\partial k}{\partial z}\right]\right. \\
\left.-K(k) \frac{\partial}{\partial z}\left(\frac{1}{p}+\frac{b}{p k^{2}}\right)-\left(\frac{1}{p}+\frac{b}{p k^{2}}\right)\left(\frac{E(k)-\left(1-k^{2}\right) K(k)}{k\left(1-k^{2}\right)}\right) \frac{\partial k}{\partial z}\right\}
\end{array}\right.
\end{aligned}
$$

After calculation and arrangement of expressions (13) and (14) we find:

$$
\left\{\begin{array}{l}
\ddot{x}=\alpha E(k)+\beta(E(k)-K(k))+\gamma K(k)+\delta\left(E(k)-\left(1-k^{2}\right) K(k)\right) \\
\ddot{z}=\eta E(k)+\mu(E(k)-K(k))+\psi K(k)+\xi\left(E(k)-\left(1-k^{2}\right) K(k)\right)
\end{array}\right.
$$

So:

$$
\left\{\begin{array}{l}
\ddot{x}=\left(\gamma-\beta-\delta\left(1-k^{2}\right)\right) K(k)+(\alpha+\beta+\delta) E(k) \\
\ddot{z}=\left(\psi-\mu-\xi\left(1-k^{2}\right)\right) K(k)+(\eta+\mu+\xi) E(k)
\end{array}\right.
$$

With:

$$
\left\{\begin{array}{l}
\alpha=-4 G \cdot \lambda_{0} \cdot a \times \frac{\partial}{\partial x}\left(\frac{b}{p \cdot k^{2}}\right)=4 G \lambda_{0} a\left[\frac{4 \cdot b a\left(z^{2}+(x+a) a\right)}{k^{4} p^{5}}\right] \\
\beta=-4 G \cdot \lambda_{0} \cdot a \times\left(\frac{b}{p \cdot k^{3}}\right) \times \frac{\partial k}{\partial x}=-4 G \lambda_{0} a\left(\frac{2 b a\left(a^{2}-x^{2}+z^{2}\right)}{p^{5} k^{4}}\right) \\
\gamma=+4 G \cdot \lambda_{0} \cdot a \times \frac{\partial}{\partial x}\left(\frac{1}{p}+\frac{b}{p \cdot k^{2}}\right)=-4 G \cdot \lambda_{0} \cdot a \times \frac{1}{k^{4} p^{3}}\left[4 a b+k^{2}(x+a)\left(k^{2}-b\right)\right] \\
\delta=+4 G \cdot \lambda_{0} \cdot a \times\left(\frac{1}{p}+\frac{b}{p \cdot k^{2}}\right) \times\left(\frac{1}{k\left(1-k^{2}\right)}\right) \times \frac{\partial k}{\partial x}=4 G \lambda_{0} a\left(\frac{2 a\left(p k^{2}+b p\right)\left(a^{2}-x^{2}+z^{2}\right)}{p^{6} k^{4}\left(1-k^{2}\right)}\right)
\end{array}\right.
$$

and

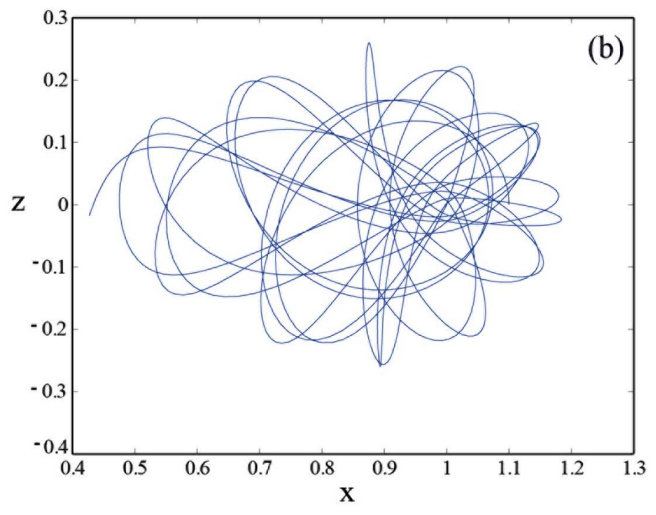

$$
\left\{\begin{array}{l}
\eta=-4 G \cdot \lambda_{0} \cdot a \times \frac{\partial}{\partial z}\left(\frac{b}{p \cdot k^{2}}\right)=-4 G \cdot \lambda_{0} \cdot a \times\left(\frac{b \cdot z}{k^{2} \cdot p^{3}}\right) \\
\mu=-4 G \cdot \lambda_{0} \cdot a \times\left(\frac{b}{p \cdot k^{3}}\right) \times \frac{\partial k}{\partial z}=4 G \lambda_{0} a\left(\frac{b z}{p^{3} k^{2}}\right)=-\eta \\
\psi=4 G \cdot \lambda_{0} \cdot a \times \frac{\partial}{\partial z}\left(\frac{1}{p}+\frac{b}{p \cdot k^{2}}\right)=4 G \lambda_{0} a\left(\frac{z}{p^{3}}\left(\frac{b}{k^{2}}-1\right)\right) \\
\xi=4 G \cdot \lambda_{0} \cdot a \times\left(\frac{1}{p}+\frac{b}{p \cdot k^{2}}\right) \times\left(\frac{1}{k\left(1-k^{2}\right)}\right) \times \frac{\partial k}{\partial z}=-4 G \lambda_{0} a\left(\frac{\left(p k^{2}+b p\right) z}{p^{4} k^{2}\left(1-k^{2}\right)}\right)
\end{array}\right.
$$

Finally:

$\left\{\begin{array}{l}\ddot{x}=A K(k)+B E(k) \\ \ddot{z}=C K(k)+D E(k)\end{array}\right.$

With:

$$
\begin{aligned}
& A=\left(\gamma-\beta-\delta\left(1-k^{2}\right)\right) \\
& B=(\alpha+\beta+\delta) \\
& C=\left(\psi-\mu-\xi\left(1-k^{2}\right)\right) \\
& D=(\eta+\mu+\xi)
\end{aligned}
$$

The system (15) represents the dynamical equations of motion of the test particle in the gravitational field generated by the inhomogeneous ring.

These equations are coupled and highly nonlinear, they require then a numerical resolution.

\section{NUMERICAL INTEGRATION}

The system (15) could be worked out by a perturbative or numerical method. We adopted, in a first time, the last one to investigate this way.

To gain deep insight about the dynamical behavior of the test particle in the field of the inhomogeneous ring, we have to solve the system (15). In this system of differential equations, the unknown variables are $\mathrm{x}$ and $\mathrm{z}$. We derive some curves in (xoz) plane.

For different values of $b$, we test many initial conditions about $v_{0 z}$ and $v_{0 x}$ separately.

This allows us to deduce the gravitational influence on the dynamical behavior of the test particle.

\subsection{Effect of $b$ on The Critical Value of $v_{0 z}$}

Fig. (4.a): $x_{0}=1,1 ; z_{0}=0 ; v_{0 x}=0 ; v_{0 z}=2 ; b=0$ 

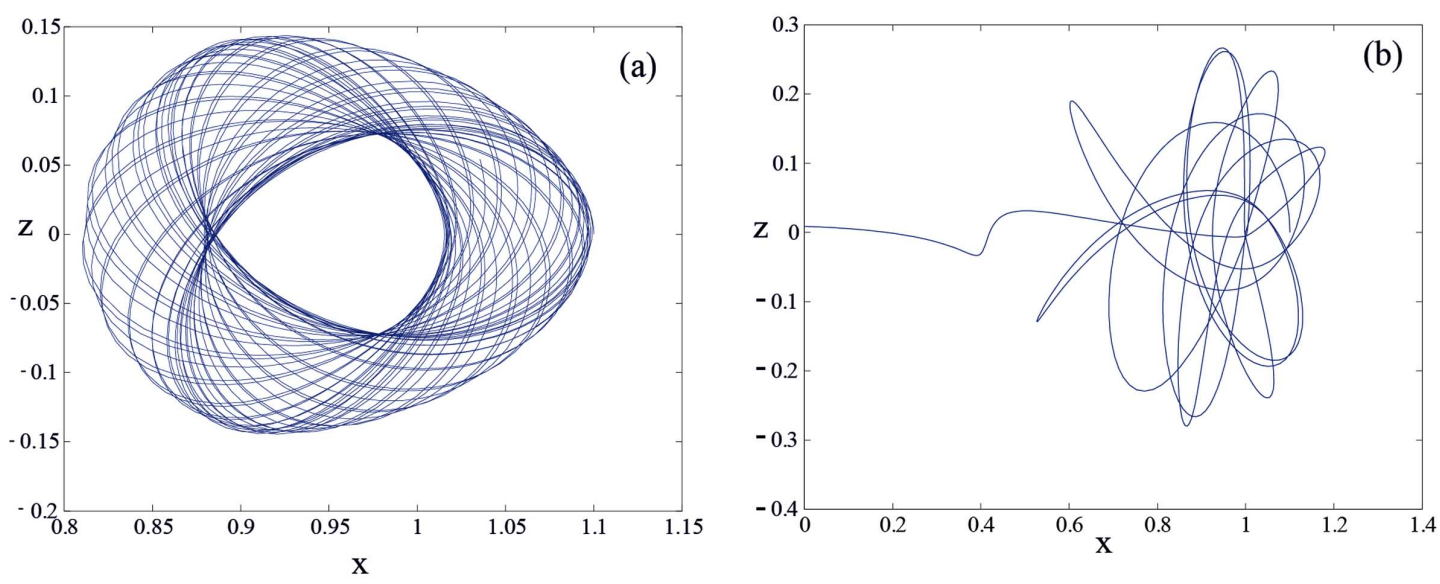

Fig. (5). Curve in (xoz) plane for $b=1: v_{0 z}=3$ and $v_{0 z}=4,62$.
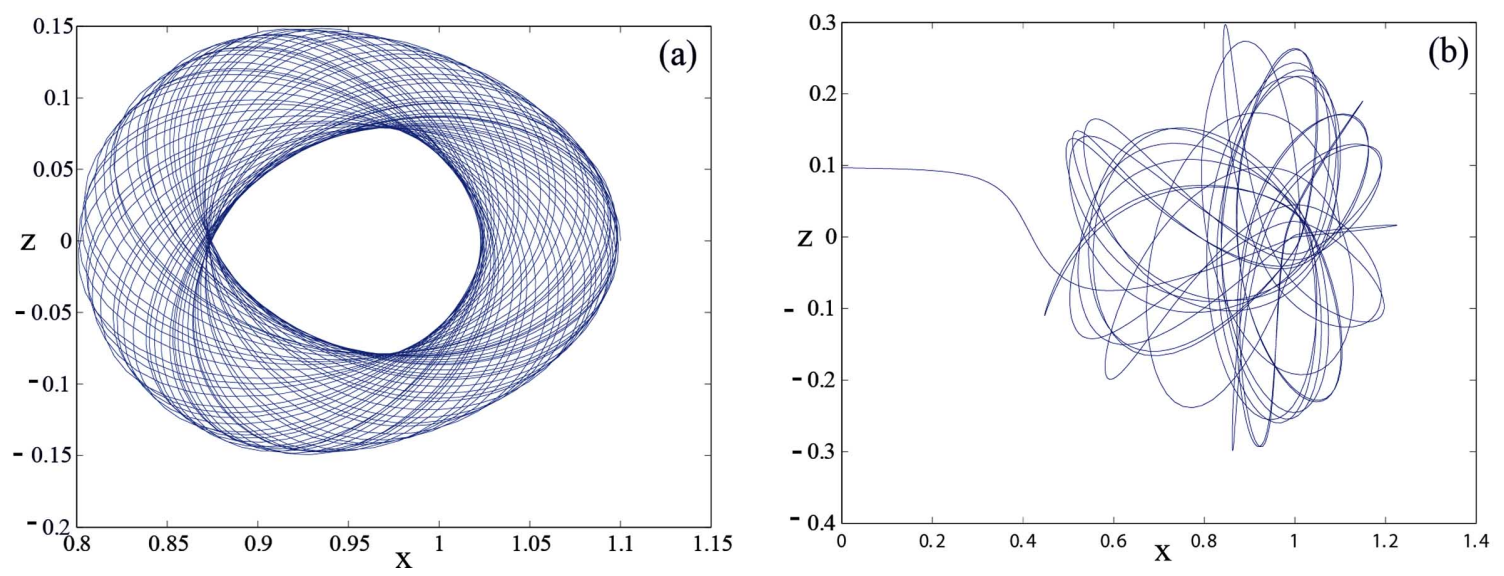

Fig. (6). Curve in (xoz) plane for $b=2: v_{0 z}=4$ and $v_{0 z}=5,82$.
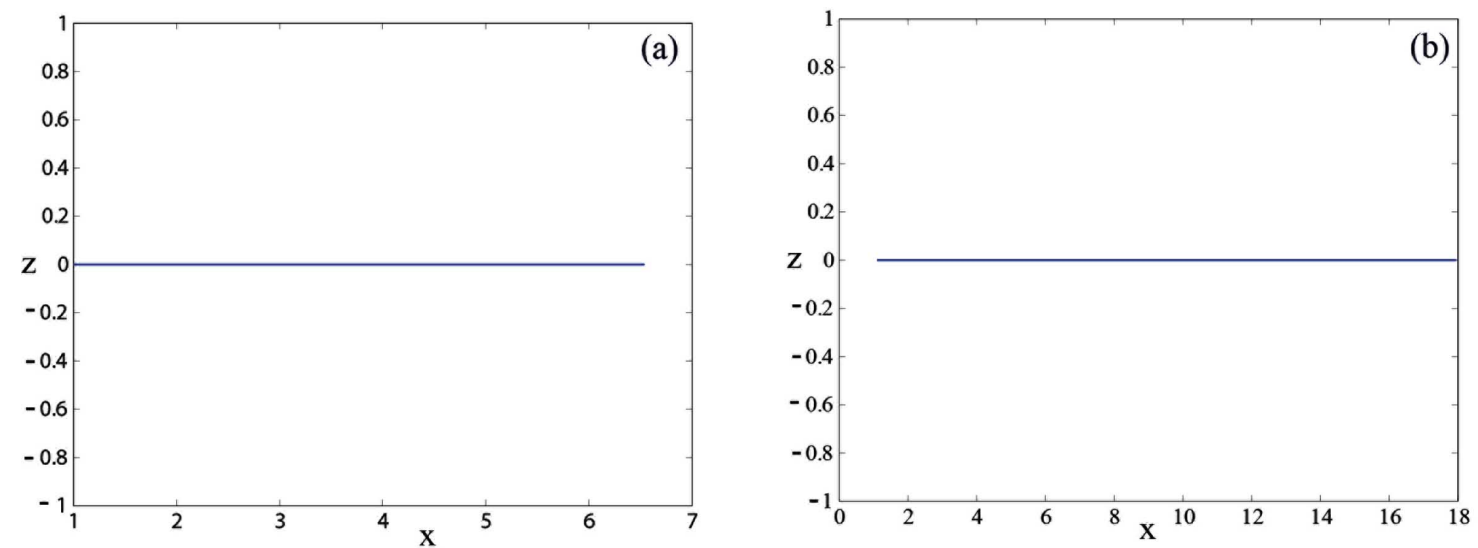

Fig. (7). Curve in (xoz) plane for $b=0: v_{0 x}=7$ and $v_{0 x}=7,8$.

Fig. (4.b): $x_{0}=1,1 ; z_{0}=0 ; v_{0 x}=0 ; v_{0 z}=3 ; b=0$

Fig. (5.a): $x_{0}=1,1 ; z_{0}=0 ; v_{0 x}=0 ; v_{0 z}=3 ; b=1$

Fig. (5.b): $x_{0}=1,1 ; z_{0}=0 ; v_{0 x}=0 ; v_{0 z}=4,62 ; b=1$

Fig. (6.a): $x_{0}=1,1 ; z_{0}=0 ; v_{0 x}=0 ; v_{0 z}=4 ; b=2$

Fig. (7.b): $x_{0}=1,1 ; z_{0}=0 ; v_{0 x}=0 ; v_{0 z}=5,82 ; b=2$

\subsubsection{Analysis of Curves}

For different values of $\mathrm{b}$, there exist a critical value $v_{0 z c}$ of $v_{0 z}$ beyond which the test particle is shifted from a

bounded state to a free state. The Table 1 gives an overview of this situation. We notice that the value of $v_{0 z c}$ grow with that of $b$, this is due to the fact that when the density is important, the escape becomes more difficult.

\subsection{Effect of $b$ on the Critical Value of $v_{0 x}$}

Fig. (8.a): $x_{0}=1,1 ; z_{0}=0 ; v_{0 x}=7 ; v_{0 z}=0 ; b=0$

Fig. (8.b): $x_{0}=1,1 ; z_{0}=0 ; v_{0 x}=7,8 ; v_{0 z}=0 ; b=0$

Fig. (9.a): $x_{0}=1,1 ; z_{0}=0 ; v_{0 x}=9,5 ; v_{0 z}=0 ; b=1$

Fig. (9.b): $x_{0}=1,1 ; z_{0}=0 ; v_{0 x}=9,6 ; v_{0 z}=0 ; b=1$ 

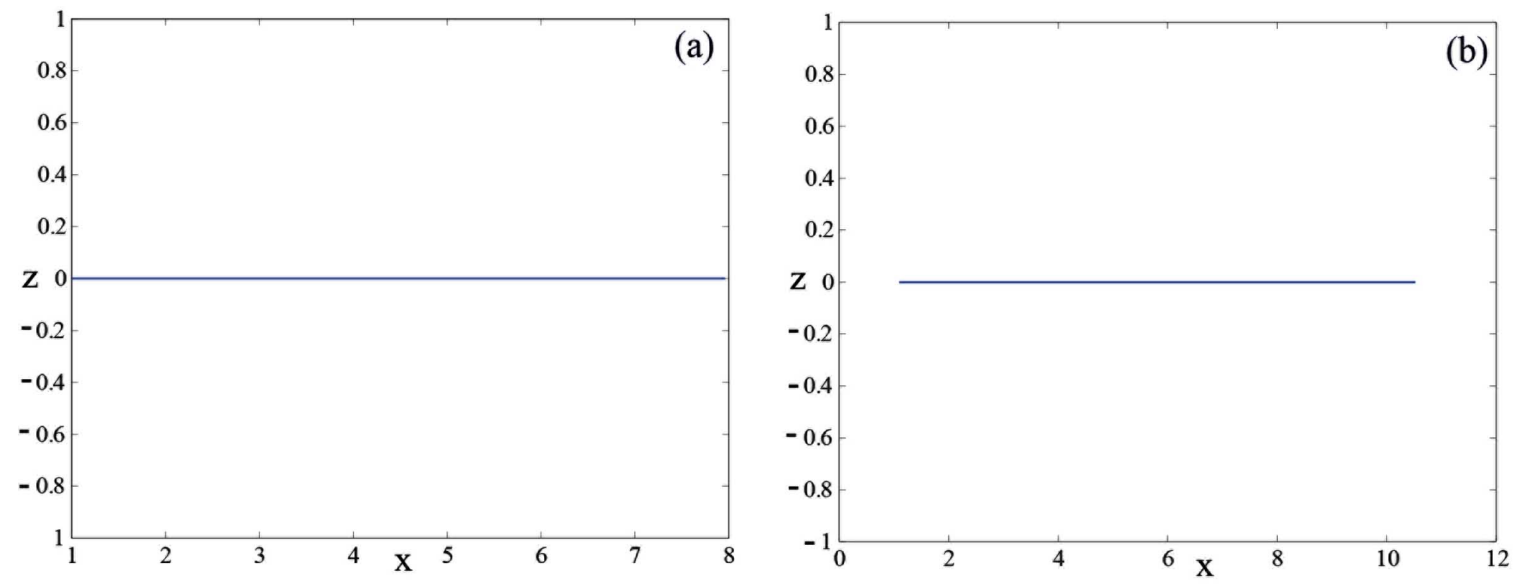

Fig. (8). Curve in (xoz) plane for $b=1: v_{0 x}=9,5$ and $v_{0 x}=9,6$.

Table 1. Effect of $b$ on the Critical Value of $V_{0 z}$

\begin{tabular}{|c|c|c|c|}
\hline Value of $\mathbf{b}$ & $\mathbf{0}$ & $\mathbf{1}$ & $\mathbf{2}$ \\
\hline \hline Critical value of $\boldsymbol{V}_{0 z}$ & 3 & 4,6 & 5,8 \\
\hline \hline
\end{tabular}

Table 2. Effect of $b$ on The Critical Value oF $V_{0 x}$

\begin{tabular}{|c|c|c|c|}
\hline Value of $\mathbf{b}$ & $\mathbf{0}$ & $\mathbf{1}$ & $\mathbf{2}$ \\
\hline \hline Critical value of $\mathrm{v}_{0 \mathrm{x}}$ & 7,42 & 9,6 & infinite \\
\hline
\end{tabular}

\subsubsection{Analysis of Curves}

For different values of $\mathrm{b}$, there exist a critical value $v_{0 x c}$ of $v_{0 x}$ beyond which the test particle is shifted from a collision state to a free state. The Table 2 give an overview of this situation.

We notice that the value of $v_{0 x c}$ grow with that of $\mathrm{b}$, this is due to the fact that when the density is important the escape becomes more difficult.

\section{CONCLUSION}

In this work we gave a new idea about the anisotropic mass distribution of a body bent into a circular shape, as in the ring of Saturn. The non-homogenous distribution of density is consistent with the photographical exploration made by many missions to Saturn like Cassini-Huygens. We established then the analytical expression in term of elliptical integrals of first and second kind, of the potential generated by that distribution. For this profile of density, we found many kind of orbits depending on the initial conditions. After reaching these results, we explored the gravitational behaviour of a test particle in the field of a ring fixed in space. This was studied by Hamiltonian formulation. In a next future, we plan to study the case of a rotating ring.

\section{CONFLICT OF INTEREST}

The author confirms that this article content has no conflicts of interest.

\section{ACKNOWLEDGEMENT}

Declared none.

\section{REFERENCES}

[1] Iorio L, Lichtenegger HIM, Ruggiero ML, Corda C. Phenomenology of the Lense-Thirring effect in the solar system. Astrophys Space Sci 2011; 331(2): 351-95.

[2] Iorio L. Classical and relativistic node precessional effects in WASP-33b and perspectives for detecting them. Astrophys Space Sci 2011; 331(2): 485-96.

[3] Iorio L. The post-Newtonian mean anomaly advance as further post-Keplerian parameter in pulsar binary systems. Astrophys Space Sci 2007; 312(3-4): 331-5.

[4] Lin-Sen Li. parameterized post-newtonian orbital effect in extrasolar planets. Astrophys Space Sci 2012; [Epub ahead of print].

[5] Iorio L. Are we far from testing general relativity with the transitting extrasolar planet HD 209458b 'Osiris'? New Astron 2007; 11(7): 490-4.

[6] Iorio L. Classical and relativistic long-term time variations of some observables for transiting exoplanets. Mon Not R Astron Soc 2011; 411(1): 167-83.

[7] Fred AC, Gregory L. Relativistic effects in extrasolar planetary systems. Int J Mod Phys D 2006; 12: 2133-40.

[8] Ioannis H, Spiros P. Satellite orbit perturbations in a dusty Martian atmosphere. Acta Astronaut 2012; 72: 27-37.

[9] Ioannis H, Omiros R, Vasile M. Yukawa-type potential effects in the anomalistic period of celestial bodies. Astrophys Space Sci 2011; 332(1): 107-13.

[10] Lin-Sen L. Influence of the gravitational radiation damping on the time of periastron passage of binary stars. Astrophys Space Sci 2011; 334(1): 125-30.

[11] Iorio L. Orbital perturbations due to massive rings. Earth Moon Planets 2012; 108(3-4): 189-217.

[12] Iorio L. Dynamical determination of the mass of the Kuiper Belt from motions of the inner planets of the Solar system. Mon Not R Astron Soc 2007; 375(4): 1311-4.

[13] Rawal JJ, Ramadurai S. Are there rings around the sun? Earth Moon Planets 2012; 108(2): 95-9.

[14] Vashkovyak MA, Vashkovyak SN. Force function of a slightly elliptical gaussian ring and its generalization to a nearly coplanar system of rings. Solar Syst Res 2012; 46(1): 69-77.

[15] MacRobert TM. Spherical harmonics. London: Methnen and Co. LTD 1927.

[16] Kellogg OD. Foundations of potential theory. New York: Dover 1953.

[17] Riaguas A, Elipe A, Lara M. Periodic orbits around natural elongated bodies. Celestial Mech Dyn Astron 1999; 73: 169.

[18] Elipe A, Lara M. A simple model for the chaotic motion around (433) eros. J Astron Sci 2003; 51: 391.

[19] Werner RA, Scheeres DJ. Exterior gravitation of a polyhedron derived and compared with harmonic and mascon gravitation representations of asteroid 4769 Castalia. Celestial Mech Dyn Astron 1997; 65: 313.

[20] Werner RA. The gravitational potential of a homogeneous polyhedron. Celestial Mech Dyn Astron 1994; 59: 253.

[21] Bartczak P, Breiter S. Double material segment as the model of irregular bodies. Celestial Mech Dyn Astron 2003; 86:131. 
[22] Bartczak P, Breiter S, Jusiel P. Ellipsoids, material points and material segments. Celestial Mech Dyn Astron 2006; 96: 31.

[23] Najid NE, Elourabi E, Zegoumou M. Potential generated by a massive inhomogeneous straight segment. Res Astron Astrophys 2011; 11(3): 345-52.

[24] Lass H, Blitzer L. The gravitational potential due to uniform disks and rings. Celestial Mech Dyn Astron 1983; 30(3): 225-8.
[25] Broucke RA, Elipe A. The dynamics of orbits in a potential field of a solid circular ring. Regul Chaotic Dyn 2005; 10: 129-43.

[26] Krough FT, Ng EW, Snyder WV. The gravitational field of a disk. Celestial Mech Dyn Astron 1982; 26: 395-405.

[27] Abramowitz M, Stegun IA. Handbook of Mathematical Functions. Natl Bur Stand Appl Math Ser 1965; 55.

Received: April 21, 2012

Revised: May 19, 2012

Accepted: June 25, 2012

(C) Najid et al.; Licensee Bentham Open.

This is an open access article licensed under the terms of the Creative Commons Attribution Non-Commercial License (http://creativecommons.org/licenses/by-nc/3.0/) which permits unrestricted, non-commercial use, distribution and reproduction in any medium, provided the work is properly cited. 